\title{
HERRAMIENTAS PARA LA GESTIÓN DE PROCESOS DE NEGOCIO Y SU RELACIÓN CON EL CICLO DE VIDA DE LOS PROCESOS DE NEGOCIO: UNA REVISIÓN DE LITERATURA
}

\section{BUSINESS PROCESS MANAGEMENT TOOLS AND ITS RELATION TO BUSINESS PROCESS LIFE CYCLE: A LITERATURE REVIEW}

\author{
Ernesto A. Galvis-Lista', Mayda Patricia González-Zabala²
}

Fecha de recepción: 30 de abril de 2014

Fecha de aprobación: 24 de septiembre de 2014

Referencia: E.A. Galvis-Lista, M.P. González-Zabala. (2014). Herramientas para la gestión de procesos de negocio y su relación con el ciclo de vida de los procesos de negocio: Una revisión de literatura. Ciencia e Ingeniería Neogranadina, 24 (2), pp. 37 - 55

\section{RESUMEN}

Este artículo presenta una revisión sobre la oferta de herramientas de tecnología de información, TI, para la gestión de procesos de negocio (Business Process Management - BPM). Para esto, se desarrolla una conceptualización de las etapas del ciclo de vida de los procesos de negocio y se realiza una descripción de las herramientas de TI desde una perspectiva funcional. Luego, se realiza un mapeo para ubicar las herramientas de TI en cada una de las fases del ciclo de vida. Este trabajo se constituye en una referencia para los profesionales que desarrollan iniciativas de BPM, así como para investigadores, profesores y estudiantes interesados en el tema. La principal contribución del trabajo es la conceptualización de las etapas del ciclo de vida de BPM y la identificación de herramientas de TI que soportan cada una de estas.

Palabras Clave: gestión de procesos de negocio, herramientas de TI para BPM, ciclo de vida de los procesos de negocio.

\section{ABSTRACT}

This paper presents a review of the wide range of information technology (IT) tools for Business Process Management (BPM). First, a conceptualization of the stages of the business processes life cycle was developed and the IT tools were described from a functional perspective. Then, a mapping of IT tools in each the phases of the life cycle of BPM was done. This work constitutes

1. Ingeniero de Sistemas, MSc. Profesor Asociado. Facultad de Ingeniería, Universidad del Magdalena, Santa Marta D.T.C.H., Colombia, egalvis@ unimagdalena.edu.co.

2. Ingeniera de Sistemas, PhD. Profesora Asociada, Facultad de Ingeniería, Universidad del Magdalena, Santa Marta D.T.C.H., Colombia, mpgonzalez@unimagdalena.edu.co 
a reference for professionals responsible for developing BPM initiatives, as well as researchers, teachers, and students interested in the subject. The main contribution of this work is the conceptualization of the stages of the BPM lifecycle and the identification of IT tools that support each of these stages.

Keywords: Business Process Management (BPM), IT tools for BPM, business process lifecycle.

\section{INTRODUCCIÓN}

En la actualidad, las organizaciones enfrentan muchos retos que han emergido a partir del fenómeno de la globalización. Para hacer frente a competidores ubicados en diversas regiones del mundo y responder rápidamente a las cambiantes condiciones del mercado, las organizaciones de diversos sectores le han prestado más atención y han invertido mayores recursos en la Gestión de Procesos de Negocio o Business Process Management (BPM) [1]. Este interés se debe a que la BPM es una forma de mejorar la visibilidad que se tiene del negocio y de dotar a las organizaciones con habilidades y herramientas que les permitan responder rápidamente a los cambios [2]. En este sentido, la BPM es un tema de gran interés para las comunidades académicas y de práctica de los campos de la administración y la computación. El amplio despliegue que ha tenido se debe a que es altamente relevante desde una perspectiva práctica y, además, ofrece retos para la investigación y la innovación en varios campos del conocimiento [3].

Las ideas que motivan y sustentan este desarrollo científico y tecnológico son dos: (1) los procesos de negocio son el instrumento clave para organizar las actividades realizadas en una organización y entender sus interrelaciones para poder mejorarlos; y (2) los Sistemas y Tecnologías de Información (STI) son un elemento esencial para soportar el desarrollo de las actividades de una organización $y$, por ende, para la gestión efectiva de sus procesos [4]. En este sentido, la BPM incluye conceptos, métodos y técnicas para el diseño, administración, configuración, acuerdo y análisis de los procesos de negocio [3].

Los beneficios que obtienen las organizaciones al implementar la BPM son: (1) mejor entendimiento y visibilidad de los procesos, (2) mejora en el manejo de las excepciones y los errores, (3) ahorro de tiempo y reducción de costos por el incremento en la eficiencia de las operaciones, (4) mejora en el desempeño de los empleados, y (5) posibilidad de mejora de los procesos con base en evidencias obtenidas por el monitoreo de la ejecución de los mismos [5-7]. Para lograrlos, las organizaciones deben asumir un enfoque centrado en los procesos, en el que se integren, en colaboración directa y responsabilidad conjunta, los responsables de las operaciones del negocio y los responsables del soporte de TI [8].

Laimplementación de laBPMenunaorganización debe estar orientada por algún modelo de ciclo de vida de los procesos de negocio. Esto permite mantener la coherencia en las acciones a ejecutar, así como un mayor entendimiento de la relación entre el resultado de la implementación y los objetivos organizacionales [3]. Además, al tener claridad sobre el soporte de herramientas de $\mathrm{TI}$ requerido en cada una de las fases del ciclo de vida, los profesionales encargados de liderar la implementación de la BPM pueden organizar 
mejor todos los procesos de desarrollo o adquisición de tecnología de acuerdo con el alcance que pretendan lograr. En este orden de ideas, este artículo presenta la descripción de varias herramientas de $\mathrm{TI}$ de soporte a la BPM, así como un análisis de su ubicación dentro del ciclo de vida de los procesos de negocio.

Este artículo ofrece una visión global y sintética sobre el tema, lo cual es útil para los profesionales de la gestión y de la informática que, estando involucrados enlaimplementación de una iniciativa de BPM en una organización, requieran conocer la gama de tecnologías aplicables en su contexto de práctica. También resulta útil para estudiantes y profesores que requieran tener una visión sintética del alcance de la BPM y sus tecnologías de soporte.

Lo que sigue del artículo está estructurado de la siguiente manera: la sección uno presenta una aproximación conceptual al ciclo de vida de los procesos de negocio, necesaria para identificar el soporte tecnológico requerido en cada una de las fases. En la sección dos se hace una descripción de cada una de las herramientas de soporte para BPM, se identifican las principales funcionalidades, los beneficios de su uso, y algunos ejemplos de productos disponibles en el mercado. La sección tres presenta el resultado del análisis sobre el tipo de soporte tecnológico requerido en cada una de las fases del ciclo de vida de los procesos de negocio. Por último, en la sección cuatro se presentan las conclusiones obtenidas al realizar este trabajo, y finalmente, las referencias utilizadas en la revisión de literatura.

\section{CICLO DE VIDA DE LOS PROCESOS DE NEGOCIO}

El primer paso para comprender el rol que juegan las tecnologías de soporte a la BPM es entender el ciclo de vida de los procesos de negocio. Por tal razón, es necesario dar cuenta de diversas propuestas para la estructura de este ciclo de vida que han sido publicadas en la literatura científica. En tal sentido, la Tabla 1 presenta los nombres de las fases utilizados en 16 propuestas de ciclo de vida.

Como se observa en la Tabla 1, se identificaron 41 denominaciones diferentes para las fases del ciclo de vida de los procesos de negocio. Lo anterior indica que existen coincidencias exactas en la denominación de algunas fases, evidenciando un amplio grado de consenso entre los investigadores. Por ejemplo, la fase de Diseño de procesos aparece ocho veces, la fase de Configuración de procesos aparece 6 veces y las fases de Diagnóstico de procesos, Implementación de procesos, Adopción de procesos y Ejecución de procesos, aparecen cuatro veces.

Por otra parte, se identificó una coincidencia semántica en varias de las fases identificadas. Por ejemplo se identificaron las fases denominadas de «Diagnóstico de Procesos» que tienen coincidencia en el propósito a las fases denominadas de «Evaluación de Procesos». También se encontraron diferentes niveles de detalle en la especificación de las fases. Por ejemplo, se encontró una fase denominada «Análisis y Diseño de Procesos» [10] y otra denominada «Análisis y Diseño» [4], que agrupa lasacciones que otros ciclos devida separanenlas fases de «Análisis» y «Diseño». Adicionalmente, se identificaron denominaciones que utilizaban términos diferentes a los tradicionales, como la fase de «Definición y alineación de procesos» [17] que indica que los procesos que han sido identificados deben quedar especificados de forma rigurosa y deben estar alineados a las metas estratégicas y operacionales del negocio, es decir, en el ámbito de acción del 
Tabla 1. Propuestas de modelos de ciclo de vida de procesos de negocio.

\begin{tabular}{|l|c|}
\hline Fases del Ciclo de Vida de los Procesos & Referencia \\
\hline $\begin{array}{l}\text { Captura y definición de procesos - Reingeniería de proceso - Implementación de procesos - Mejora continua de } \\
\text { procesos. }\end{array}$ & {$[9]$} \\
\hline Análisis y diseño de procesos - Modelado de procesos - Implementación de procesos. & {$[10]$} \\
\hline Diseño de procesos - Configuración de procesos - Adopción de procesos - Diagnóstico de procesos. & {$[11]$} \\
\hline Diseño de procesos - Configuración de sistemas - Adopción de procesos - Diagnóstico y mejora de procesos. & {$[13]$} \\
\hline $\begin{array}{l}\text { Diseño de procesos - Configuración de procesos - Ejecución de procesos - Control de procesos - Diagnóstico } \\
\text { de procesos. }\end{array}$ & {$[14]$} \\
\hline $\begin{array}{l}\text { Análisis de procesos - Diseño de procesos - Implementación de procesos - Ejecución de procesos - Monitoreo } \\
\text { de procesos - Evaluación de procesos. }\end{array}$ & {$[15]$} \\
\hline Estrategia de procesos- Modelado de procesos - Implementación de procesos - Control de procesos. & {$[16]$} \\
\hline $\begin{array}{l}\text { Diseño de procesos - Configuración de procesos - Ejecución de procesos - Control de procesos - Diagnóstico } \\
\text { de procesos. }\end{array}$ & {$[17]$} \\
\hline $\begin{array}{l}\text { Identificación de necesidades de procesos - Determinación de cambios en procesos - Definición y alineación de } \\
\text { procesos - Implementación e integración de procesos. }\end{array}$ & {$[18]$} \\
\hline $\begin{array}{l}\text { Análisis de procesos - Diseño de procesos - Configuración de procesos - Adopción de procesos - Evaluación de } \\
\text { procesos. }\end{array}$ & {$[19]$} \\
\hline $\begin{array}{l}\text { Descubrimiento de procesos - Diseño de procesos - Configuración de procesos - Integración de servicios - } \\
\text { Despliegue de procesos - Ejecución de procesos - Monitoreo de procesos - Mejora de procesos. }\end{array}$ & {$[20]$} \\
\hline Diseño de procesos - Configuración de procesos - Adopción de procesos - Diagnóstico de procesos. & {$[21]$} \\
\hline Diseño - Modelado - Ejecución - Monitoreo. & {$[22]$} \\
\hline $\begin{array}{l}\text { Estrategia de procesos - Arquitectura de procesos - Propiedad de procesos - Medición de procesos - Mejora de } \\
\text { procesos. }\end{array}$ & {$[4]$} \\
\hline Análisis y diseño - Configuración - Adopción - Evaluación. & {$[23]$} \\
\hline Identificación - Modelado - Análisis - Mejora - Implementación - Ejecución - Monitoreo. & {$[12$} \\
\hline
\end{tabular}

análisis y diseño de los procesos. La síntesis de las diferentes denominaciones para las fases del ciclo de vida de los procesos de negocio se presenta en la Tabla 2.

A partir de la síntesis presentada en la Tabla 2 , se puede decir que el ciclo de vida de los procesos de negocio, como se muestra en la Figura 1, consiste en fases relacionadas y organizadas en una estructura que, como lo argumentan Van der Aalst [3] y Weske [4], no implica una dependencia temporal o un orden estricto en el que deban ejecutarse. En lugar de esto, el ciclo de vida de los procesos de negocio puede entenderse como un proceso evolutivo e incremental. Este enfoque permite que las organizaciones inicien la implementación de iniciativas de BPM sin tener que asumir una etapa de aprestamiento demasiado exigente en términos técnicos, financieros y organizacionales. Además, delinea un camino

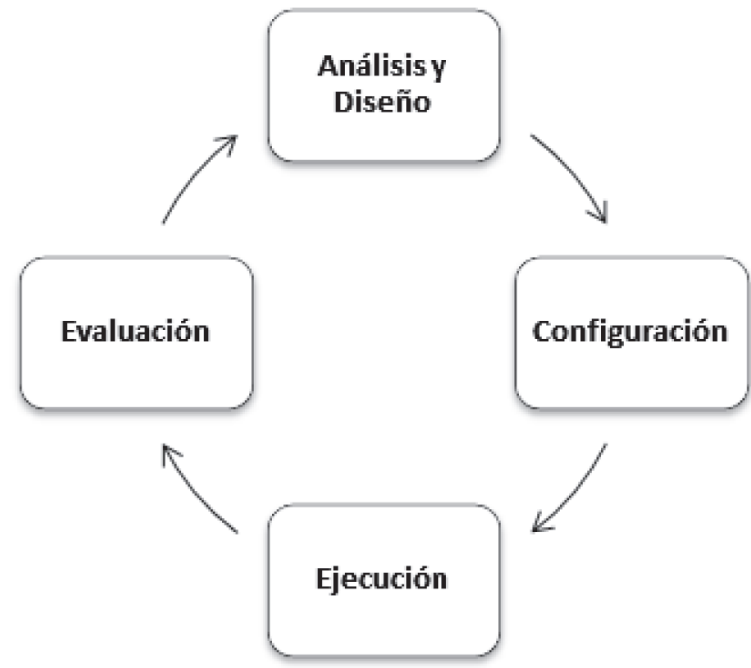

Figura 1. Ciclo de vida de los procesos de negocio. Fuente: Elaboración propia a partir de [4].

de mejora continua y sostenible para los procesos de la organización [12]. 
Tabla 2. Síntesis de cuatro fases del ciclo de vida de los procesos de negocio.

\begin{tabular}{|c|c|c|}
\hline Objetivo & $\begin{array}{l}\text { Denominación } \\
\text { común }\end{array}$ & Fases propuestas por los autores \\
\hline $\begin{array}{l}\text { Entender el estado actual de los } \\
\text { procesos de la organización y } \\
\text { especificar las características que } \\
\text { deberían tener para asegurar } \\
\text { objetivos relacionados con la } \\
\text { eficiencia, eficacia y efectividad. }\end{array}$ & Análisis y Diseño & $\begin{array}{l}\text { Análisis - Análisis de procesos - Análisis y diseño - } \\
\text { Análisis y diseño de procesos - Arquitectura de } \\
\text { procesos - Captura y definición de procesos - } \\
\text { Definición y alineación de procesos - } \\
\text { Descubrimiento de procesos - Diseño - Diseño de } \\
\text { procesos - Estrategia de procesos - Identificación - } \\
\text { Identificación de necesidades de procesos - } \\
\text { Modelado - Modelado de procesos - Propiedad de } \\
\text { procesos - Reingeniería de proceso. }\end{array}$ \\
\hline $\begin{array}{l}\text { Configurar, implementar y } \\
\text { desplegar el proceso diseñado en } \\
\text { el entorno de ejecución que utiliza } \\
\text { la organización. }\end{array}$ & Configuración & $\begin{array}{l}\text { Configuración - Configuración de procesos - } \\
\text { Configuración de sistemas - Despliegue de } \\
\text { procesos - Implementación - Implementación de } \\
\text { procesos - Implementación e integración de } \\
\text { procesos - Integración de servicios. }\end{array}$ \\
\hline $\begin{array}{l}\text { Ejecutar y hacer seguimiento y } \\
\text { control a las múltiples instancias } \\
\text { de los procesos que son } \\
\text { necesarias para el desarrollo de } \\
\text { las actividades y propósitos } \\
\text { organizacionales. }\end{array}$ & Ejecución & $\begin{array}{l}\text { Adopción - Adopción de procesos - Control de } \\
\text { procesos - Ejecución - Ejecución de procesos. }\end{array}$ \\
\hline $\begin{array}{llll}\text { Identificar las fortalezas y } & \text { y } \\
\text { debilidades } & \text { del proceso con el } \\
\text { propósito de identificar y } & \text { de } \\
\text { especificar mejoras que puedan } \\
\text { ser implementadas. }\end{array}$ & Evaluación & $\begin{array}{l}\text { Determinación de cambios en procesos - } \\
\text { Diagnóstico de procesos - Diagnóstico y mejora de } \\
\text { procesos - Evaluación - Evaluación de procesos - } \\
\text { Medición de procesos - Mejora - Mejora continua de } \\
\text { procesos - Mejora de procesos - Monitoreo - } \\
\text { Monitoreo de procesos. }\end{array}$ \\
\hline
\end{tabular}

Como se mencionó antes, el objetivo de la fase de análisis y diseño es entender el estado actual de los procesos de la organización y especificar las características que deberían tener para asegurar objetivos relacionados con la eficiencia, eficacia y efectividad. En esta fase se deben identificar y modelar los procesos que existen en la organización [24]. Estos procesos pueden estar formalmente identificados y documentados, como en un sistema de gestión de la calidad, o simplemente pueden estar establecidos dentro de la cultura y política de la organización sin que medie una representación formal del mismo. Para lograr la participación de la mayor parte de los involucrados en el proceso en la identificación y modelado del mismo, se recomienda la utilización de notaciones estándar como la Notación de Modelado de Procesos de Negocio o BPMN Business Process Modeling Notation. Una vez modelado el proceso, tal y como se ejecuta en la organización, se procede a evaluarlo con el fin de identificar mejoras que deben ser incorporadas al proceso dentro de las especificaciones de diseño [17]. Para esto se pueden utilizar técnicas de trabajo colaborativo, así como métodos de análisis cuantitativo de procesos y herramientas de simulación. Estas últimas permiten que los participantes del proceso observen la ejecución paso a paso de modo que se puedan detectar comportamientos indeseados. [4]. 
Al tener el proceso diseñado y validado se puede proceder a configurar, implementar y desplegar el proceso diseñado en el entorno de ejecución que utiliza la organización. Para esto se deben incorporar políticas y procedimientos a las prácticas tradicionales de la organización, y se puede utilizar el soporte de un sistema software que permita su implementación y su ejecución [12]. En caso de optar por la incorporación de un sistema software, se debe realizar la evaluación y selección de la herramienta más adecuada de acuerdo con las características del proceso, y también se debe enriquecer la especificación del diseño del proceso con detalles técnicos necesarios para la configuración y despliegue del proceso en el software seleccionado [4]. La configuración del sistema software debe incluir las interacciones de los empleados con el sistema y la integración con otros sistemas software utilizados por la organización.

Esto último puede implicar trabajo en construcción de aplicaciones que puedan enlazar sistemas software de uso específico con el sistema de gestión de procesos de negocio, o el diseño e implementación de una arquitectura orientada a servicios que se encargue de ese tipo de interacciones. Adicionalmente, dependiendo de las características del proceso, se deben tener en cuenta aspectos relacionados con el manejo de transacciones [4]. Una vez implementado el proceso con todas sus características, se deben realizar validaciones y pruebas para asegurar la calidad. Aquí pueden utilizarse técnicas para la realización de pruebas propias de la Ingeniería de Software, como pruebas de unidad (a nivel de actividades de proceso), pruebas de rendimiento e integración (a nivel del proceso). Por último, el proceso implementado debe ser desplegado en el entorno de producción.
La fase de ejecución del proceso de negocio requiere que la configuración esté completa. Esta fase comprende todo el tiempo en el que los procesos de negocio se encuentran en tiempo de ejecución. Aquí se crean tantas instancias del proceso como sea necesario para cumplir con los objetivos de la organización. El rol del sistema software de soporte a la gestión de procesos de negocio es controlar activamente la ejecución de cada una de las instancias del proceso de acuerdo con lo definido en el modelo. En esta fase se recopilan datos sobre la ejecución del proceso, incluyendo el estado de cada una de las instancias en cualquier instante de tiempo. Adicionalmente, estos datos recopilados pueden ser visualizados utilizando diversas técnicas que faciliten el monitoreo de las instancias del proceso [19]. Todos los datos recopilados serán utilizados en la fase de evaluación del proceso.

En la fase de evaluación se utilizan los datos recopilados en la fase de ejecución para identificar las fortalezas y debilidades del proceso. Esta evaluación debe dar como resultado la especificación de mejoras para el proceso y su implementación [12]. La fase de evaluación debe tomar como línea base datos de la ejecución del proceso antes de la implementación, los cuales pueden obtenerse de datos históricos o de los resultados de simulaciones. Adicionalmente, se recomienda que el análisis del proceso involucre diversas perspectivas desde las cuales se puedan emitir juicios, por ejemplo, la perspectiva operacional del proceso, la perspectiva financiera, y, la siempre muy importante, perspectiva de los usuarios del proceso. En esta fase se pueden utilizar instrumentos como tableros de comando que permitan presentar la síntesis de los resultados del proceso en diversas perspectivas. Sumado 
a esto, es importante mencionar que este ciclo de vida genera numerosos artefactos y actividades en diferentes niveles de la organización que requieren organizarse y administrarse de la mejor manera. Por ejemplo, el problema de almacenar y recuperar toda la documentación relacionada con los procesos y su implementación técnica debe ser resuelto utilizando procedimientos y herramientas que faciliten el acceso de las personas a estos recursos.

De la descripción de cada fase del ciclo de vida se pueden identificar un conjunto de resultados esperados, que se presentan en la Tabla 3. Estos resultados son necesarios y suficientes para lograr el objetivo de la fase. Además, la forma particular que toman estos resultados depende de las características de los procesos que se gestionan y de la forma en que se lleva a cabo el ciclo de vida en cada organización. Por ejemplo, el monitoreo del proceso, que hace parte de la fase de ejecución, podría materializarse por medio de herramientas de gestión como cuadros de mando o indicadores claves de desempeño.

Por último, es necesario expresar que durante todo el ciclo de vida las personas de la organización asumen uno o varios roles relacionados con la gestión de los procesos de negocio. Estos roles se encargan de hacer que el ciclo se desarrolle de acuerdo con los objetivos de la organización. En este sentido, las personas, dependiendo de sus niveles de responsabilidad, experiencia y conocimiento podrían asumir alguno de los siguientes roles: Director de Procesos (Chief Process Officer), Ingeniero de Procesos de Negocio (Business Process Engineer), Diseñador de Procesos (Process Designer), Participante de proceso (Process Participant), Trabajador de conocimiento (Knowledge Worker), Responsable de Proceso (Process Responsible), Arquitecto de software (Software Architect), y Desarrollador de software (Software Developer) [25-27].

Tabla 3. Descripción de las fases del ciclo de vida de los procesos de negocio.

\begin{tabular}{|c|c|}
\hline Fase & Resultados \\
\hline Análisis y Diseño & $\begin{array}{l}\text { - Identificación y modelado de los procesos existentes en la organización. } \\
\text { Identificación de las mejoras a ser implementadas. } \\
\text { Diseño del proceso mejorado. } \\
\text {. Documentación. }\end{array}$ \\
\hline Configuración & $\begin{array}{l}\text { Implementación del proceso mejorado. } \\
\text { Evaluación de herramientas software que podrían apoyar el proceso. } \\
\text { Nuevo diseño del proceso donde se relacionan las actividades, los actores y las } \\
\text { herramientas de apoyo (aspectos técnicos). } \\
\text { - Implementación técnica del proceso. } \\
\text { Resultados de las validaciones y de las pruebas de calidad. } \\
\text { Documentación. }\end{array}$ \\
\hline Ejecución & $\begin{array}{l}\text { Definición de las instancias del proceso. } \\
\text { Datos sobre la ejecución del proceso. } \\
\text { Monitoreo del proceso. }\end{array}$ \\
\hline Evaluación & $\begin{array}{l}\text { Especificación de mejoras para el proceso y su implementación. } \\
\text { Resultados del proceso desde múltiples perspectivas (usuario, aspectos operacionales, } \\
\text { financieros, etc.). }\end{array}$ \\
\hline
\end{tabular}




\section{HERRAMIENTAS PARA LA GESTIÓN DE PROCESOS DE NEGOCIO}

Para realizar el análisis propuesto en este trabajo se requiere tener una descripción de los diferentes tipos de herramientas para la gestión de procesos de negocio, de modo que la clasificación de las herramientas respecto de las fases del ciclo de vida sea interpretada de forma clara. Por lo tanto, en esta sección se identifican y describen, para cada herramienta, aspectos como sus principales características funcionales, los beneficios potenciales que brindan a la organización y los nombres de algunos productos ofertados en el mercado.

\subsection{ANÁLISIS DE PROCESOS DE NEGOCIO}

Las herramientas de Análisis de Procesos de Negocio (Business Process Analysis - BPA) son componentes clave para las iniciativas de mejora de procesos y la implementación de programas de BPM. Estas proporcionan los medios para realizar un análisis detallado de los procesos de una organización. Las funcionalidades principales que ofrecen estas herramientas son las de modelado, simulación y publicación de los procesos. Su uso se hace prioritariamente por los ingenieros de procesos y a los participantes de procesos, pues ellos tienen el conocimiento tácito concerniente al proceso a analizar y a la forma en que se puede modelar y mejorar. Sin embargo, tendencias actuales proponen incorporarlas en las prácticas cotidianas de la organización, o como se ha denominado, el "uso de BPA por las masas" [28].

Por otra parte, las herramientas de BPA proporcionan más flexibilidad para los participantes de procesos al agregarles dimensiones adicionales a los modelos de proceso. Aquí se pueden agregar la representación de recursos físicos y humanos, restricciones normativas, y riesgos y problemas asociados directamente a una actividad o a todo el proceso [29]. También, algunas herramientas proporcionan funcionalidades para la generación de reportes que permiten que toda la información relativa al proceso sea publicada y compartida electrónicamente. Por otra parte, dado que la mayoría de herramientas de BPA tienen un repositorio compartido para los modelos, es posible hacer análisis del impacto de los cambios en las organizaciones y en los roles, con base en las interrelaciones de sus modelos [30]. Porúltimo, es importante aclarar que las herramientas de BPA complementan a las suites de BPM al permitir modelar los procesos en múltiples niveles y con gran detalle, incluyendo el mapeo de las relaciones.

Dos ejemplos de este tipo de herramientas son BlueWorksLive de IBM [31-32], ARISalig de Software AG [33-34] e Igrafx [35]. Las dos primeras introducen en su funcionalidad el concepto de BPM social, permitiendo la participación de todos los miembros de la organización, así como la colaboración interorganizacional. Las suites de BPM también incluyen la funcionalidad de las herramientas de BPA.

\subsection{DESCUBRIMIENTO AUTOMATIZADO DE PROCESOS DE NEGOCIO}

Las herramientas de Descubrimiento Automatizado de Procesos de Negocio (Automated Business Process Discovery ABPD) permiten «descubrir» los procesos de negocio con base en el análisis de todos los registros electrónicos dejados por los participantes del proceso en cada una de las herramientas de tecnología de información que se utilizan en la organización para dar 
soporte el proceso [36]. Estas herramientas asumen un enfoque de «Bottom - Up» para la construcción del modelo de proceso, es decir, que toman hechos detallados de las instancias del proceso para tratar de abarcar todos los matices que toma su ejecución. Además, se enriquece la descripción con información estadística detallada de cuán a menudo se ejecutan diferentes rutas o caminos del proceso, cuánto tiempo toman y qué variaciones se dan entre diferentes usuarios 0 grupos de usuarios, entre otros aspectos. Este enfoque, alternativo al tradicional enfoque de trabajo colaborativo con las personas, ofrece ventajas como la identificación de ineficiencias en los procesos, que permanecen tácitas para los participantes, la identificación de patrones de acción como mejores prácticas, y mayor eficiencia en el proceso de descubrimiento y documentación de los procesos.

Para implementar una herramienta de descubrimiento automático de procesos, las organizaciones deben desplegar y ejecutar una herramienta para la recopilación de datos sobre las acciones de los usuarios, en donde, a manera de registro de transacciones (log), se almacenen todos los datos que permitan visualizar la manera en que se ejecutan los procesos en la organización [37]. Esta herramienta de recopilación de datos registrará, por ejemplo, las transacciones realizadas por los usuarios en el sistema de administración de recursos empresariales o ERP, la interacción que se tiene con los clientes y proveedores a través de los sistemas de administración de relaciones con los clientes o CRM y de administración de la cadena de suministros o SCM.

Con todos los datos recopilados se realiza una «reconstrucción» de la ejecución de cada instancia del proceso, en donde se puede identificar la secuencia de actividades representada en un grafo que se denomina «Cadena de Proceso Manejado por Eventos» o Event-driven process chain (EPC). En procesos complejos, con un elevado número de instancias, la representación de cada una es ensamblada con las demás, generadas a partir de las otras instancias del proceso, para obtener una colección que es sintetizada en una imagen «promedio» de su ejecución [38]. Adicionalmente, estas herramientas pueden identificar y «descubrir» relaciones organizacionales, es decir, responder preguntas como ¿Quién trabaja con quién y cómo? [3839]. En síntesis, este tipo de herramientas le permiten a las organizaciones modelar los procesos actuales 0 «As-Is» como realmente se ejecutan, pues se obtienen a partir de la evidencia recopilada de múltiples instancias del proceso [40-41]. Un ejemplo de la aplicación de estas herramientas es el trabajo de Peters, Dedene y Houck [42], que presenta la utilización de herramientas de este tipo para el análisis de los procesos de solicitudes y reclamaciones médicas en el sistema de salud de los Estados Unidos.

Por otra parte, las principales limitaciones y retos que enfrentan este tipo de herramientas están relacionadas con el manejo de flujos de control complejos, duplicados, la consistencia interna del modelo generado, y la granularidad y especificidad de los modelos generados [43]. Sin embargo, también se encuentran desarrollos investigativos que tratan con problemas complejos como el de la utilización de registros de actividades (activity logs) no estructurados como fuente para el descubrimiento de modelos de procesos de negocio [44].

Dentro de los productos disponibles en el mercado se destacan el ofrecido por Pallas Athena, compañía holandesa que según 
Sinur y Hill [45] tiene la mejor tecnología y visualización para el descubrimiento automatizado de procesos. Otro producto, con mayor posicionamiento en el mercado, es el comercializado como servicio por la compañía Fujitsu y que hace parte de la suite denominada Fujitsu Interstage BPM. Por último, es importante mencionar el producto denominado ARIS Process Performance Manager de Software AG, que contiene, entre otras, la funcionalidad de ABPD. Un análisis detallado de las técnicas y herramientas utilizadas actualmente se puede encontrar en el trabajo de Turner, Tiwari y Olaiya [46].

\subsection{MONITOREO DE ACTIVIDADES DE NEGOCIO}

El concepto de monitoreo de actividades de negocio o Business Activity Monitoring (BAM) fue propuesto originalmente por investigadores de la empresa de investigación y consultoría Gartner Inc. La idea central es la recopilación, organización, análisis y visualización de datos obtenidos en tiempo real, acerca de las actividades ejecutadas en un proceso de negocio. En palabras de MsCoy [47], el BAM proporciona acceso en tiempo real a indicadores críticos del desempeño del negocio para mejorar la velocidad y la efectividad de las operaciones. El BAM beneficia a las organizaciones en la medida en que permite tomar de forma rápida decisiones con mejor información sobre el desempeño de los procesos de negocio y, más rápidamente, identificar y resolver problemas durante la ejecución del proceso [48].

En la práctica, el monitoreo de las actividades del negocio se centra en tomar mediciones de una gran variedad de métricas sobre la ejecución del proceso de negocio, como volumen de instancias, duración, costos, errores y condiciones especiales. Las herramientas de BAM mejoran el desempeño de las organizaciones porque aseguran que los procesos de negocio operan como se esperaba, aseguran que los procesos de negocio cumplen con los compromisos y propósitos de la organización, y reducen la latencia entre el momento en que se realiza la medición del desempeño y el momento en que se toman las decisiones [49]. Esencialmente, las herramientas de BAM intentan proveer a las organizaciones lo que los instrumentos de aeronavegación proporcionan a los pilotos de las aeronaves: datos en tiempo real sobre el comportamiento de las operaciones.

Las soluciones de BAM se conforman esencialmente por cuatro componentes: eventos de negocio, es decir, eventos que son importantes en el contexto de la ejecución de una actividad del negocio; actividades del negocio, es decir, actividades incluidas en un proceso de negocio para brindar un servicio o funcionalidad requerida para cumplir con una necesidad específica de la organización; métricas, es decir, mediciones sobre propiedades específicas de un actividad del negocio que puede utilizarse para monitorear el desempeño de la operación; e indicadores clave de desempeño (KPI), que son métricas utilizadas para medir el progreso de un objetivo organizacional a nivel estratégico [50].

Al revisar la oferta de herramientas de BAM, se encuentra que las funcionalidades comunes que ofrecen son: captura instantánea de datos sobre indicadores clave de desempeño en diferentes niveles del proceso; presentación sintética de los datos de desempeño en tableros de instrumentos (dashboard) configurables por el usuario y con diferentes tipos de visualización de los datos; notificaciones automáticas sobre problemas o posibles violaciones a políticas y niveles de desempeño establecidos para el 
proceso de negocio; y generación de reportes sobre el comportamiento y tendencias de los indicadores de desempeño del proceso de negocio [51-54].

Estas funcionalidades parecen similares a las ofrecidas por herramientas de inteligencia de negocios (Business Intelligence - BI). Sin embargo, es necesario hacer la distinción entre las dos, pues, aunque están ubicadas en la misma categoría de herramientas de análisis del negocio, tienen una diferencia fundamental sobre el origen de los datos analizados. Las herramientas de inteligencia de negocios también tienen que ver con el entendimiento y la optimización del desempeño del negocio, pero la diferencia es que éstas se enfocan en el análisis de datos históricos que son utilizados para explorar, utilizando técnicas de minería de datos, el comportamiento que se ha tenido en períodos anteriores y asi poder identificar tendencias. Por otra parte, las herramientas de BAM tratan con los datos de lo que está sucediendo actualmente en el negocio, es decir, en tiempo real [53]. Tres ejemplos de este tipo de herramientas son Business Monitor de IBM [53], Business Activity Monitoring de Oracle [52] y BusinessFactor de TIBCO Software Inc [54].

\subsection{SISTEMAS DE ADMINISTRACIÓN DE REGLAS DE NEGOCIO}

Las reglas de negocio son la codificación de políticas, normas, leyes y mejores prácticas que son utilizadas por una organización para tomar decisiones. Una regla de negocio requiere una estructura formal en donde se representan elementos atómicos de la lógica del negocio y las acciones que deberían ejecutarse en ciertas circunstancias [55]. Comúnmente, estas reglas están embebidas en la especificación de procedimientos y en las herramientas de Tecnología de Información que soportan la ejecución de los procesos de negocio [56]. En el contexto de la Gestión de Procesos de Negocio tradicional, es común observar modelos de proceso en donde cada una de las reglas que determinan el comportamiento de la organización son codificadas e incluidas en forma de estructuras de decisión dentro de la notación del modelo. Este enfoque genera problemas relacionados con el mantenimiento y la evolución de los modelos de proceso, pues el cambio de una política, por ejemplo la política de descuentos a clientes, implica la modificación del modelo para cumplir con las nuevas reglas.

Por otra parte, al utilizar un enfoque de Gestión de Reglas de Negocio, las reglas no se implementan dentro del modelo, sino que son mantenidas de forma separada para ser utilizadas y modificadas sin generar cambios en los modelos de proceso de negocio. De esta manera se dota a la organización de mayor agilidad y capacidad de adaptación a los frecuentes cambios del entorno [57]. En este sentido, la incorporación del enfoque de reglas de negocio en BPM produce varios beneficios que van más allá de la agilidad y la facilidad de cambio. Separar las reglas de las estructuras de decisión de los modelos de proceso permite que estas se puedan cambiar en tiempo real y sin necesidad de parar la ejecución del proceso en respuesta a condiciones cambiantes. Por otra parte, se reduce el riesgo de error en la toma de decisiones al automatizar reglas que no requieren de la participación de una persona. También, se reducen costos de operación al eliminar del proceso los pasos de verificación que no generan valor. Además, se mejora el desempeño en el cumplimiento de las reglas y se proporciona trazabilidad y transparencia en la toma de decisiones [58].

Los sistemas para la administración de reglas de negocio o BRMS (Business Rule Management 
Systems - BRMSs) permiten modificaciones dinámicas y rápidas de la implementación de las políticas o normas de la organización. Los BRMS son aplicaciones software en donde se definen, simulan, almacenan, despliegan, ejecutan, monitorean y optimizan las reglas del negocio. Además, se pueden desplegar en una arquitectura orientada a servicios (Service Oriented Architecture - SOA) en donde el motor de reglas se presenta como un servicio que proporciona los resultados de la ejecución de las reglas del negocio ante una solicitud de otra aplicación que proporciona los datos de entrada necesarios para aplicar la regla [56]. Dentro de la oferta de productos disponibles en el mercado se encuentran WebSphere ILOG Business Rule Management System de IBM [59], FICO Blaze Advisor de Fair Isaac Corporation [60], y JBoss Enterprise BRMS de RedHat [61].

\subsection{SUITES DE GESTIÓN DE PROCESOS DE NEGOCIO}

En el mercado de las herramientas de soporte a BPM es común encontrar distribuidores que ofrecen en un solo paquete un conjunto de herramientas integradas en lo que se ha denominado Suite de Gestión de Procesos de Negocio (Business Process Management Suites - BPMSs). Sin embargo, hay dos elementos esenciales que debe tener una suite de BPM: un entorno de modelado y simulación de procesos, y un motor de ejecución de procesos.

En el entorno de modelado y simulación se brinda la funcionalidad para que los modelos de procesos puedan ser implementados y probados antes de entrar en uso. Por otra parte, el motor de ejecución se encarga de automatizar y hacer seguimiento a la ejecución de todas las instancias del proceso implementado, es decir, es el corazón de cualquier solución de BPM.
Además, utiliza herramientas para integrar el proceso de negocio con interfaces o servicios de aplicaciones y sistemas existentes. Ejemplos de BPMS son Oracle Business Process Management Suite [62], IBM Business Process Manager [63], y BizAgi BPM suite [64].

\section{CLASIFICACIÓN DE LAS TECNOLOGÍAS DE GESTIÓN DE PROCESOS DE NEGOCIO EN EL CICLO DE VIDA DE LOS PROCESOS}

Para iniciar esta sección es conveniente recapitular y sintetizar lo dicho hasta el momento. Por esta razón, en la Tabla 4 se presenta un sumario de las principales funcionalidades y los productos de ejemplo de los cuatro tipos de herramientas descritos en la sección anterior. Es de aclarar que en esta tabla se excluyó al tipo de herramienta denominado BPMS pues, como se dijo antes, éstas integran las características funcionales de las demás.

Una vez descritos el ciclo de vida de los procesos de negocio y las tecnologías de soporte para la gestión de procesos de negocio, se debe realizar un mapeo para identificar cómo las funcionalidades ofrecidas por cada tipo de herramienta brinda soporte a una o varias fases del ciclo de vida de los procesos de negocio. La Tabla 5 ubica para cada fase del ciclo de vida los tipos de herramientas que brindan soporte para su ejecución.

Las herramientas de Análisis de Procesos de Negocio se utilizan en las fases de análisis y diseño porque permiten que los usuarios participen en la construcción de los modelos, también brindan soporte a la evaluación pues sirven como referente o línea base para la comparación del desempeño obtenido con el desempeño esperado. Las herramientas de Descubrimiento Automatizado de Procesos de 
Tabla 4. Características funcionales y ejemplo de los tipos de herramientas para BPM.

\begin{tabular}{|c|c|c|}
\hline Herramienta & Funcionalidades & Productos de Ejemplo \\
\hline BPA & $\begin{array}{l}\text { - Analizar detalladamente los procesos organizacionales } \\
\text { - Modelar, simular y publicar procesos en múltiples } \\
\text { niveles } \\
\text { - Representar los recursos físicos y humanos, las } \\
\text { restricciones normativas, los riesgos y los problemas. } \\
\text { - Generar reportes } \\
\text { - Compartir información acerca de los procesos. } \\
\text { - Permitir la participación de los miembros de la } \\
\text { organización }\end{array}$ & $\begin{array}{l}\text { BlueWorksLive de IBM } \\
\text { ARISalig de Software } \\
\text { AG } \\
\text { - Igrafx } \\
\text {. Savvion Process } \\
\text { Modeler } \\
\text { - Intalio Designer, TIBCO } \\
\text { Business Studio }\end{array}$ \\
\hline ABPD & $\begin{array}{l}\text { Registrar las transacciones efectuadas en cada } \\
\text { proceso } \\
\text { - Analizar los registros electrónicos dejados por los } \\
\text { actores del proceso en cada una de las herramientas } \\
\text { de soporte del proceso. } \\
\text { - Generar de estadísticas sobre la ejecución de rutas o } \\
\text { caminos del proceso, tiempos de ejecución, variaciones } \\
\text { entre usuarios. } \\
\text { - Identificar ineficiencias de los proceso, patrones de } \\
\text { acción } \\
\text { - Identificar y descubrir relaciones organizacionales }\end{array}$ & $\begin{array}{l}\text { Fujitsu Interstage BPM } \\
\text { ARIS Process } \\
\text { Performance Manager } \\
\text { de Software AG }\end{array}$ \\
\hline BAM & $\begin{array}{l}\text { Recopilar, organizar, analizar y visualizar datos, en } \\
\text { tiempo real, de las actividades ejecutadas del proceso } \\
\text { de negocio. } \\
\text { - Acceso en tiempo real a indicadores críticos del } \\
\text { desempeño de negocios } \\
\text { - Identificar y resolver problemas durante la ejecución de } \\
\text { los procesos } \\
\text { Calcular métricas sobre la ejecución del proceso }\end{array}$ & $\begin{array}{l}\text { Business Monitor de } \\
\text { IBM } \\
\text { Business Activity } \\
\text { Monitoring de Oracle } \\
\text { BusinessFactor de } \\
\text { TIBCO Software Inc. }\end{array}$ \\
\hline BRMS & $\begin{array}{l}\text { Modificar de forma dinámica y rápida la implementación } \\
\text { de políticas o normas de la organización. } \\
\text { Definir, simular, almacenar, desplegar, ejecutar y } \\
\text { monitorear las reglas del negocio. } \\
\text { - Permitir su despliegue en arquitecturas orientadas a } \\
\text { servicios }\end{array}$ & $\begin{array}{l}\text { FICO Blaze Advisor } \\
\text { - WebSphere ILOG } \\
\text {. JBoss Enterprise BRMS }\end{array}$ \\
\hline
\end{tabular}

Tabla 5. Relación de los tipos de herramientas para BPM con las fases del ciclo de vida de procesos de negocio.

\begin{tabular}{|c|c|c|c|c|}
\hline Herramienta & $\begin{array}{c}\text { Análisis } \mathbf{y} \\
\text { Diseño }\end{array}$ & Configuración & Ejecución & Evaluación \\
\hline BPA & $\mathbf{X}$ & & & $\mathbf{X}$ \\
\hline ABPD & $\mathbf{X}$ & $\mathbf{X}$ & & $\mathbf{X}$ \\
\hline BAM & & & $\mathbf{X}$ & $\mathbf{X}$ \\
\hline BRMS & & & $\mathbf{X}$ & \\
\hline
\end{tabular}


Negocio sirven como soporte para el análisis y diseño, la configuración y, principalmente, la evaluación pues toman todos los datos de la ejecución de los procesos para identificar tendencias, caminos frecuentes, errores comunes, entre otros. Esto permite tomar decisiones de mejora de procesos con base en evidencia. Por último, las herramientas de BAM y BRMS sirven como soporte a la ejecución de los procesos.

\section{CONCLUSIONES}

Esta revisión se ha orientado a buscar una visión sintética sobre las herramientas de TI de soporte a la gestión de procesos de negocio y su relación con el ciclo de vida de los procesos de negocio. Para esto se realizaron tres análisis: 1) identificación y descripción de las fases del ciclo de vida de los procesos de negocio, 2) identificación y descripción de herramientas de TI de soporte a la BPM, y 3) ubicación de las herramientas de $\mathrm{TI}$ identificadas dentro de las fases del ciclo de vida de los procesos de negocio.

Sobre la conceptualización realizada acerca de las fases del ciclo de vida de los procesos de negocio, se pudo apreciar que las propuestas de los autores analizados se pueden sintetizar en cuatro fases esenciales que son: análisis y diseño, configuración, ejecución y evaluación. Un aspecto importante de esta síntesis es que las fases propuestas están concebidas y configuradas como un ciclo continúo que permite mejorar los procesos desarrollados de acuerdo con las necesidades organizacionales, en lugar de actividades aisladas que se enfocan exclusivamente en ciertas funciones organizacionales.

Así mismo, la descripción que se realizó sobre las herramientas de $\mathrm{Tl}$ que brindan soporte a la BPM ofrece una panorámica de las principales características funcionales de cada una de estas, los beneficios que ofrecen, los productos desarrollados y los fabricantes que trabajan en el campo de la gestión de procesos de negocios. Con esto, se identifica que éste es un nicho importante para los profesionales en $\mathrm{Tl}$, especialmente en Colombia, en donde la apropiación de este tipo de tecnologías no ha tenido suficiente cobertura.

Sobre el tercer análisis, se presenta la relación entre las herramientas desarrolladas y las etapas del ciclo de vida de la BPM, lo cual se constituye en una guía para entender las diferentes opciones que dichas herramientas ofrecen a las organizaciones para soportar sus procesos de gestión. Esto es principalmente útil al momento de tomar decisiones sobre qué tecnología utilizar de acuerdo con los intereses y el estado de desarrollo de la organización.

En cuanto a las implicaciones prácticas de este artículo, cabe mencionar que brinda una visión sintética que puede servir de guía a los ejecutivos de las organizaciones sobre las diversas opciones que tienen para desarrollar las iniciativas de BPM de acuerdo con las metas y objetivos de la organización. También es útil en procesos de formación y capacitación en el tema, al presentar el alcance que tiene la Gestión de Procesos de Negocio en la actualidad.

Sumado a esto, es necesario declarar que el análisis realizado se limitó a las descripciones funcionales de las herramientas en forma genéricay noseprofundizóen lascaracterísticas funcionales específicas que ofrecen los productos disponibles en el mercado. En este sentido, se propone como trabajo futuro realizar una evaluación pormenorizada de los productos existentes en el mercado, a la luz de las funcionalidades necesarias para que se 
soporten las diferentes fases del ciclo de vida de los procesos de negocio. Además, se propone la realización de estudios en organizaciones de diferentes sectores de la economía para obtener evidencias empíricas de la forma en que las herramientas soportan las fases del ciclo de vida y del impacto que se genera en las organizaciones con la implementación de este tipo de herramientas.

\section{REFERENCIAS BIBLIOGRÁFICAS}

[1] Becker, J., Rosemann, M. \& von Uthmann, C. (2000). Guidelines of business process modeling. En: Business Process Management. Berlín, Alemania: Springer, pp.30-49.

[2] Serrano Gómez, L. \& Ortiz Pimiento, N. R. (2012). Una revisión de los modelos de mejoramiento de procesos con enfoque en el rediseño. Estudios Gerenciales, 28(125), pp.13-22.

[3] van der Aalst, W. M. (2013). Business process management: A comprehensive survey. ISRN Software Engineering, 2013.

[4] Weske, M. (2007). Business Process Management: Concepts, Languages, Architectures (1st ed.). Berlín, Alemania: Springer.

[5] Software AG. (2007). Getting Started with Business Process Management. Software AG Documentation.

[6] Elzinga, D. J., Horak, T., Lee, C.-Y. \& Bruner, C. (1995). Business process management: survey and methodology. Engineering Management, IEEE Transactions on, 42(2), pp.119-128.
[7] Zairi, M. (1997). Business process management: a boundaryless approach to modern competitiveness. Business Process Management Journal, 3(1), pp.64-80.

[8] Garimella, K., Lees, M. \& Williams, B. (2008). BPM basics for dummies, Software AG special edition. Hoboken, NJ, EE.UU.: Wiley.

[9] Georgakopoulos, D. \& Tsalgatidou, A. (1998). Technology and tools for comprehensive business process lifecycle management. NATO ASI SERIES $F$ COMPUTER AND SYSTEMS SCIENCES, 164, pp.356-395.

[10] Tsalgatidou, A. \& Panepistimiopolis, T. (1998). Selectioncriteria for tools supporting business process transformation for electronic commerce. Proceedings of EURO-MED NET'98, pp. 244-253.

[11] Van der Aalst, W.M.P., Hofstede, A.H.M. \& Weske, M. (2003). Business Process Management: A Survey. En W.M.P. Van der Aalst \& M. Weske (Eds.), Business Process Management, vol. 2678. Berlín, Alemania: Springer, pp.1-12.

[12] Van der Aalst, W.M.P. (2004). Business Process Management Demystified: A Tutorial on Models, Systems and Standards for Workflow Management. En J. Desel, W. Reisig, \& G. Rozenberg (Eds), Lectures on Concurrency and Petri Nets, vol. 3098. Berlín, Alemania: Springer, pp.1-65.

[13] Netjes, M., Reijers, H.A. \& van der Aalst, W.M. (2006). FileNet's BPM life-cycle support. BPM Center Report BPM-0607, BPMcenter.org. 
[14] Hrastnik, J., Cardoso, J. \& Kappe, F. (2007). The Business Process Knowledge Framework, ICEIS, 3, pp.517-520.

[15] Klückmann, J. On the way to SOABusiness processes as a guideline. ARIS Expert Paper.

[16] van derAalst, W.MP., Netjes, M. \& Reijers, H.A. (2007). Supporting the Full BPM Life-Cycle Using Process Mining and Intelligent Redesign. Contemporary issues in database design and information systems development, p.100.

[17] Williams, B. (2007). BPM: The Next Stage for Continous Process Improvement. Software AG, webMethods.

[18] Rotaru, K., Wilkin, C., Ceglowski, A. \& Churilov, L. (2008). How Critical Realism Contributes to Understanding within the Business Process Lifecycle. Systal Class Notes Module.

[19] Scheithauer, G., Wirtz, G. \& Toklu, C. (2008). Bridging the semantic gap between process documentation and process execution. En The 2008 International Conference on Software Engineering and Knowledge Engineering (SEKE'08), Redwood City, California, EE.UU.

[20] Ko, R.K.L., Lee, S.S.G. \& Lee, E.W. (2009). Business process management (BPM) standards: a survey. Business Process Management Journal, 15(5), pp.744-791.

[21] Weber, B., Sadiq, S. \& Reichert, M. (2009). Beyond rigidity-dynamic process lifecycle support. Computer Science-Research and Development, 23(2), pp.47-65.
[22] Smart, P.A., Maddern, H. \& Maull, R.S. (2009). Understanding Business Process Management: Implications for Theory and Practice. British Journal of Management, 20(4), pp.491-507.

[23] Mathiesen, P., Watson, J., Bandara, W. \& Rosemann, M. (2012). Applying Social Technology to Business Process Lifecycle Management. En F. Daniel, K. Barkaoui, S. Dustdar, W. Aalst, J. Mylopoulos, M. Rosemann, M.J. Shaw \& C. Szyperski (Eds.), Business Process Management Workshops, vol. 99. Berlín, Alemania: Springer, pp.231-241.

[24] Gramlich, C. (s.f.). Business Process Analysis. Process-oriented Information Systems.

[25] Hammer, M. (2010). What is Business Process Management? En J. vom Brocke \& M. Rosemann (Eds.), Handbook on Business Process Management 1, Berlín, Alemania: Springer, pp.3-16.

[26] Sousa, K., Mendonça, H., Lievyns, A. \& Vanderdonckt, J. (2011). Getting users involved in aligning their needs with business processes models and systems. Business Process Management Journal, 17(5), pp.748-786.

[27] Sonteya, T. \& Seymour, L. (2012). Towards an Understanding of the Business Process Analyst: An Analysis of Competencies. Journal of Information Technology Education: Research, 11, pp.43-63.

[28] Norton, D., Blechar, M. \& Jones, T. (2010). Magic Quadrant for Business Process Analysis Tools. Gartner RAS Core Research Note G. 
[29] Davies, I., Reeves, M. \& Sherman, J. (2007). Business Process Analysis Tool [FC] Program.

[30] Berecz, P. (2009). Tools of Business Process Modeling. En Proceedings of the 4th Aspects and Visions of Applied Economics and Informatics.

[31] IBM Corporation. (2012). IBM Blueworks Live, the roadmap to tackle process improvement. IBM: Somers, EE.UU.

[32] IBM Corporation. (2012) IBM Blueworks Live. Recuperado el 6 de agosto de 2012, de https://www.blueworkslive. com/\#!gettingStarted:overview

[33] Mendling, J., Recker, J.C. \& Wolf, J. (2012). Collaboration features in current BPM tools. EMISA Forum, 32, pp.48-65.

[34] Software AG. (2012). ARISalign space. Recuperado el 6 de agosto de 2012, de http://www.arisalign.com/

[35] iGrafx, LLC. (2012). iGrafx Process 2011 for Enterprise Modeling. Recuperado el 6 de agosto de 2012, de http://www.igrafx. com/products/process4em/

[36] Open Connect Systems Inc. (2007). Automated Business Process Discovery for Quantified Process Improvement. Open Connect Systems Inc.

[37] lontas. (2008). Automated Process Discovery for BPM and BPI Initiatives Technology Brief. lontas.

[38] Blickle, T. \& Hess, H. (2009). Automatic Process Discovery with ARIS Process Performance Manager (ARIS PPM). Expert Paper, IDS Scheer.
[39] Blickle, T., Hess, H., Klueckmann, J., Lees, M. \& Williams, B. (2010) Process Intelligence For Dummies ${ }^{\circledR}$, Software AG Special Edition. EE.UU.: Wiley Publishing, Inc.

[40] Fujistu America Inc. (2009). THE 'AS-IS ' AS IT REALLY IS - Process Discovery and Visualization from Fujitsu (FPC58-239401). Fujistu America Inc.

[41] Fujistu America Inc. (2009). Automated Process Discovery improves business processes at a leading photonic solutions company (FPC58-2611-01). Fujistu America Inc.

[42] Peters, E.M., Dedene, G. \& Houck, C. (2009). Business Process Discovery \& Workforce Intelligence Techniques with Healthcare Applications. En Proceedings of the 35th Conference on Operational Research Applied to Health Services, Leuneven, Bélgica.

[43] van der Aalst, W.M. (2010). Process Discovery: Capturing the Invisible. Computational Intelligence Magazine, IEEE, 5(1), pp.28-41.

[44] Kumar, R., R. Bhattacharyya, R. \& Varshneya, V. (2010). Discovering Business Process Model from Unstructured Activity Logs. Services Computing (SCC), 2010 IEEE International Conference, pp. 250257.

[45] Sinur J. \& Hill, J.B. (2010). Magic Quadrant for Business Process Management Suites. Gartner RAS Core research note, $1-24$.

[46] Turner, C.J., Tiwari, A. \& Olaiya, R. (2002) Business process mining: from theory to 
practice. Business Process Management Journal, 18(3), pp.6-6.

[47] MsCoy, D. (2002). Business Activity Monitoring: Calm Before the Storm. Recuperado el 6 de agosto de 2012, de https://www.gartner.com/doc/354283/ business-activity-monitoring-calm-storm

[48] Adams, P. (2002). What's going on? [business activity monitoring]. Manufacturing Engineer, 81(6), pp.282283.

[49] Reybaud, A.L., Moreno, J.J., Novales, R. \& Aguilar, L.J. (2005). Business Activity Monitoring y Business Rules para el Manejo de Excepciones en las Políticas en un sistema de Gestión de Procesos de Negocios. Estado del-Arte. En IV SIMPOSIO INTERNACIONAL DE SISTEMAS DE INFORMACIÓN, p.195.

[50] Amnajmongkol, S., Keen, M., Ang'ani, J., Che, Y. \& Fox, T. (2008) Business activity monitoring with WebSphere business monitor V6.1. EE.UU.:IBM International Technical Support Organization.

[51] Peyret, H. (2008). Best Practices: Business Activity Monitoring Adoption. Forrester Research, Inc,.

[52] Srivasta, P. (2009). Oracle Business Activity Monitoring. Oracle Corporation, Technical Report.

[53] Craggs, S. (2010). Using IBM BPM to Maximize the Value of Business Interactions. Creating Business Value Through Better Insight with IBM Websphere Business Monitor. Lustrator Research Limited.
[54] TIBCO Software Inc. (2011). TIBCO | Business Activity Monitoring Software. Recuperado el 6 de agosto de 2012, de http://www.tibco.com/software/ business-activity-monitoring/default.jsp.

[55] FICO Corp. (2010). Enhancing BPM with Business Rules. Fair Isaac Corporation.

[56] Meservy, T., Lee, E., Dhaliwal, J. \& Zhang, C. (2009). An Exploratory Case Study of the Benefits of Business Rules Management Systems. ICIS 2009 Proceedings.

[57] Stineman, B. (2009). Why Business Rules?: A Case for Business Users of Information Technology. IBM.

[58] Kemsley, S. (2008). Business Rules Management and Business Process Management: Turning Policies into Action. Corticon Technologies, Inc.

[59] IBM Corporation. (s.f.). IBM WebSphere ILOG - a recognized industry leader in Business Rule Management Systems (BRMS), optimization, visualization and supply chain solutions. Recuperado el 28 de agosto de 2012, de http://www01.ibm.com/software/websphere/ilog/

[60] FICO Corp. (s.f.). BRMS - Business Rules Management System - FICO ${ }^{\circledR}$ Blaze Advisor ${ }^{\circledR}$. Recuperado el 28 de agosto de 2012, de http://www.fico.com/en/ products/dmtools/pages/fico-blazeadvisor-system.aspx.

[61] Red Hat Inc. (s.f.). Red Hat - JBoss Enterprise Business Rules Management System (BRMS). Recuperado el 28 de agosto de 2012, de http://www.redhat.com/products/ jbossenterprisemiddleware/business-rules/ 
[62] Oracle (s.f.). Business Process Management Suite - Oracle. Recuperado el 28 de agosto de 2012, de http://www. oracle.com/us/technologies/bpm/suite/ overview/index.html.

[63] IBM Corporation. (s.f.). IBM - Business Process Manager | IBM. Recuperado el 28 de agosto de 2012, de http://www- 01.ibm.com/software/integration/ business-process-manager/

[64] BizAgi.(s.f.). Businessprocessmanagement, BPM, iBPM, workflow automation software - Bizagi iBPMS. Recuperado el 28 de agosto de 2012, de http://www.bizagi. com/index.php?option=com_content\&vie $\mathrm{W}=$ article\&id $=16 \& \mathrm{Itemid}=83$ 